\title{
MedienPädagogik
}

Zeitschrift für Theorie und Praxis der Medienbildung

www.medienpaed.com

ISSN 1424-3636

Jahrbuch Medienpädagogik 17:

Lernen mit und über Medien in einer digitalen Welt

Herausgegeben von Klaus Rummler, Ilka Koppel, Sandra Aßmann,

Patrick Bettinger und Karsten D. Wolf

\section{Editorial: Lernen mit und über Medien in einer digitalen Welt}

Klaus Rummler, Ilka Koppel, Sandra Aßmann, Patrick Bettinger und Karsten D. Wolf

Lernen mit und über Medien war und ist in der deutschsprachigen Medienpädagogik Inhalt und Ziel mediendidaktischer und medienerzieherischer Überlegungen und Aktivitäten. Im Fokus stehen dabei sowohl formale als auch non-formale und informelle Kontexte des Lehrens und Lernens sowie verschiedene Zielgruppen quer über alle Altersgruppen hinweg. Durch curriculare Neuerungen oder diverse Rahmenkonzepte für Bildungssettings auf Bundes-, Landes-, oder Kantonsebene wird das Thema zunehmend auch Teil anderer Fachdidaktiken und - so könnte man argumentieren - auch zu einer eigenständigen Fachdidaktik. Aus dieser Überlegung entstanden für das 17. Jahrbuch Medienpädagogik unter dem Titel «Lernen mit und über Medien in einer digitalen Welt» folgende strukturierende Themenfelder «Gestaltung von Lehren und Lernen mit Medien» sowie «Lern- und Bildungsprozesse im Kontext der Digitalität». In allen Beiträgen dieses Jahrbuchs steht das Thema Lernen im Zentrum. Mit diskutiert werden dabei auch Querschnittsfragen nach Lernen in unterschiedlich charakterisierten Kontexten wie z. B. formell - informell, oder institutionell - persönlich. Lernen mit und über Medien wird in einer thematisch breiten Perspektive sowohl anhand empirischer Projekte als auch durch grundlegende theoretische Überlegungen bearbeitet. 


\section{Themenfeld: Gestaltung von Lehren und Lernen mit Medien}

Mit dem Begriff der Mediendidaktik hat das Thema Gestaltung von Lehren und Lernen mit Medien eine lange Tradition in der Medienpädagogik mit einem engen Bezug zu «e-learning», «blended-learning», CSCL und Lernplattformen im Allgemeinen. Dies begründet dann auch die zentrale Bedeutung der Mediendidaktik im Handlungs- und Forschungsfeld Hochschuldidaktik. In diesen Bereichen stellt sich die Frage nach aktuellen Erkenntnissen sowie nach erfolgreichen oder gescheiterten Projekten, Ansätzen und den jeweiligen Ursachen. Dieser Bereich ist traditionell von Wirksamkeitsstudien geprägt, die sich mit der - nur auf den ersten Blick trivialen - Frage beschäftigen, wie Lernen mit Medien erfolgreich gestaltet werden kann. «Erfolgreich» ist in diesem Sinne ein Container-Begriff, der je nach Kontext ausgelegt werden kann und muss. Gleiches gilt für Begriffe wie «Effizienz», «Effektivität» oder «Mehrwert». Deutlich wird, dass erst die Verbindung von Technologie- und Organisationsentwicklungskonzepten eine qualitativ hochwertige, effektive Nutzung digitaler Medien in Schule, Hochschule und weiteren Lernsettings erzielen kann, und somit weniger das «Was» sondern stärker das «Wie» Aufschluss über Gelingensbedingungen geben kann. Hinzu kommt die zunehmende Bedeutung informeller Lernanlässe, die besonders durch die Omnipräsenz und die intensive Nutzung digitaler Medien und verschiedenster Online-Plattformen in den vergangenen Jahren einen deutlichen Schub bekamen. Fragen der Gestaltung von Lehren und Lernen sind damit längst nicht mehr nur allein auf die (hoch-)schulischen Praktiken in Lehr-/Lernsettings begrenzt, sondern betreffen vielmehr Bildungsorganisationen und -institutionen als ganze.

Für die Ausbildung von Lehrpersonen ist vor allem das Thema Lernen mit Medien bedeutsam und wird in den Beiträgen «Big Data als datenbasierte Programmierung» von Christian Swertz, «Technological Pedagogical Content Knowledge> als Leitmodell medienpädagogischer Kompetenz» von Mirjam Schmid und Dominik Petko sowie «Lehren lernen mit digitalen Medien» von Falk Scheidig behandelt. Spezifisch den Bereich Partizipation und Didaktik bearbeiten die Beiträge «Partizipative Mediendidaktik» von Kerstin Mayrberger und «Lernen mit und über Wikibooks» von Franco Rau. Perspektiven des multimodalen Lernens zeigen die Beiträge 
«Selbstreferentialität und Historisierung als Werkzeuge medialen Handelns im bildungswissenschaftlichen Kontext» von Katharina Wedler und der Beitrag «Leseförderung 4.0?» von Maik Philipp auf.

Drei Beiträge behandeln aktuelle Fragen zur Gestaltung von Lehrmitteln: «Zwischen traditionellem Schulbuch und hybridem Lehrmittel» von Alexandra Totter, Julia Häbig, Daniela Müller-Kuhn und Enikö Zala-Mezö; «Lernen mit immersiver Virtual Reality: Didaktisches Design und Lessons Learned» von Josef Buchner und Diane Aretz sowie «Veränderung von Einstellungen und Verhaltensdeterminanten mittels videobasierter Narrationen mit parallelen Erklärungen» von Christina Sick.

Dem Thema Tablets im Unterricht widmen sich folgende drei Beiträge, indem sie aus Schulprojekten resümierend berichten: «Digitale Medien in der Grundschullehrerbildung» von Horst Niesyto und Thorsten Junge, «Tablets an Beruflichen Gymnasien: Gelingensfaktoren für die Integration mobiler Endgeräte im Schulunterricht» von Kerstin Mayrberger und Kathrin Galley sowie «Lernunterstützung mit digitalen Unterrichtsmaterialien» von Richard Böhme und Meike Munser-Kiefer.

Einblicke in fachdidaktische Projekte im Themenfeld Lernen mit Medien geben die Beiträge «Inszenierungsmöglichkeiten eines mediengestützten Sportunterrichts» von Mareike Thumel, Anja Schwedler-Diesener, Steffen Greve, Jessica Süßenbach, Florian Jastrow und Claus Krieger; «Medienkompetenzförderung im Lehramtsstudium der Goethe-Universität» von Natalie Kiesler sowie «Lehren und Lernen mit und über Medien in Kooperation von Schule, Hochschule und Museen» von Daniel Autenrieth, Claudia Baumbusch und Anja Marquardt.

\section{Themenfeld: Lern- und Bildungsprozesse im Kontext der Digitalität}

Die Beiträge des zweiten thematischen Clusters befassen sich mit der Frage, wie sich Lern- und Bildungsprozesse angesichts der Ubiquität und der gesellschaftsprägenden Rolle digital-medialer Strukturen verändern (müssen). Auch diese Perspektive hat eine vergleichsweise lange Tradition in der Medienpädagogik. Auf den ersten Blick offenbart sich hier ein 
anhaltender Diskurs um grundbegriffliche Setzungen: So zeigen sich Aushandlungen um die Konturierung und Abgrenzung der Begriffe Medienerziehung, Medienkompetenz (und deren Förderung in pädagogischinstitutionellen Settings) sowie Medienbildung - einerseits verstanden als fachdidaktisches Handlungskonzept in der Schule, andererseits als Veränderung lebensweltlicher Orientierung unter Bedingungen von (digitaler) Medialität - miteinander. Hinzu kommt, dass der sprachliche Bedeutungsgehalt von Medienbildung regional unterschiedlich ist und vor allem in Österreich und in der Schweiz im Kern eher die Medienerziehung (im Verständnis des deutschen Fachdiskurses) bezeichnet als den theoretischen Rahmen zum Medienhandeln in der Alltagswelt oder Veränderungen von Selbst- und Welthaltungen im Kontext von Medialität. Geprägt sind Fragen des Lernens im Kontext der Digitalität zudem von programmatischen Vorschlägen, die an der Schnittstelle von Wissenschaft und Bildungspolitik operieren und inhaltliche Setzungen aushandeln, die curricularen Niederschlag finden sollen. Hierbei fällt auf, dass Diskurse um Bildung und Digitalisierung zwar recht prominent in der Öffentlichkeit verhandelt werden, den eigentlichen Entwicklungen in Technik, Gesellschaft und Bildungspraxis teilweise aber hinterherhinken. Insofern wäre beispielsweise zu fragen, ob bildungspolitische Initiativen wie das KMK-Papier in Deutschland (KMK - Kultusministerkonferenz 2016), Schule 4.0 in Österreich (Bundesministerium für Bildung, Wissenschaft und Forschung 2017), oder Lehrplan 21 in der Deutschschweiz (Deutschschweizer Erziehungsdirektoren-Konferenz - D-EDK 2014) und die dadurch angestossenen Prozesse die Herausforderungen der Digitalisierung nachhaltig gut abbilden, oder eher zeitverzögerte Reaktionen sind, und wie dann wiederum eine kritische Medienpädagogik mittelfristig damit umgehen müsste. In dieser Diskussion kommt neuerdings die Frage nach der Informatik - bzw. der Didaktik der Informatik - hinzu, mit Implikationen, was denn aktuell über bestimmte mediale Erscheinungsformen wie soziale Online-Netzwerke oder Robotik auf der informatischen Seite zu lernen sei. Ein Beispiel für einen entsprechenden interdisziplinären Systematisierungsversuch ist das Dagstuhl- und Frankfurt-Dreieck (Brinda u. a. 2016; Brinda u. a. 2019).

Als Teile dieses Themenfelds berichten Alexander Repenning, Nora Anna Escherle und Anna Lamprou von «Die ersten 1000: Computational 
Thinking als obligatorische Ausbildung für Primarschullehrpersonen in der Schweiz». Patrick Bettinger, Saskia Draheim, Simon Meier und Ellen Witte liefern in diesem Themenfeld «Eine materiell-diskursive Perspektive auf Lernprozesse in Makerspaces und FabLabs» mit dem Titel «Making» the subject».

Die Beiträge «Bildung in der digitalen Welt: Über Wirkungsannahmen und die soziale Konstruktion des Digitalen» von Michael Kerres sowie «Resonanzstrategien im Umgang mit Beschleunigung und Digitalisierung» von Jana Wienberg und Klaus Buddeberg widmen sich spezifisch dem Thema Digitalisierung und Lernen. Maike Altenrath, Christian Helbig und Sandra Hofhues fragen in ihrem Beitrag nach diskurs- und machtpolitisch hervorgerufenen Deutungshoheiten unter dem Titel «Digitalisierung und Bildung in Programmatiken und Förderrichtlinien Deutschlands und der EU». Einen (fach-)didaktischen Blick auf Lernplattformen werfen die Beiträge «Pädagogisch-Didaktische Herausforderungen bei der Entwicklung von digitalen Lernumgebungen in der laborbasierten Lehre» von David Schepkowski, Martin Burghardt und Peter Ferdinand; «Visualisierung und Didaktisierung digitaler Archivbestände» von Katharina Gallner-Holzmann; «Lernen mit und über digitale Medien im Sachunterricht» von Carmen Kunkel, Markus Peschel sowie «Mittendrin statt nur dabei» von Elke Schlote, Daniel Klug und Klaus Neumann-Braun. Der Beitrag «Überlegungen zum Lernen mit und über Medien im Zeitalter der Digitalisierung» von Heinz Moser (Keynote) rundet das Themenfeld ab.

Die Beiträge im 17. Jahrbuch Medienpädagogik unter dem Titel «Lernen mit und über Medien in einer digitalen Welt» wurden auf der gleichnamigen Herbsttagung der Sektion Medienpädagogik der DGfE am Donnerstag, 26. und Freitag, 27. September 2019 an der Pädagogischen Hochschule Zürich als Präsentationen diskutiert (vgl. Rummler und Koppel 2019). Teile der Tagung wurden per Video-Stream an die Pädagogische Hochschule Weingarten geleitet, wo dieser Tagungsteil als Weiterbildungsveranstaltung stattfand.

Die Beiträge in diesem Band wurden zunächst als Abstracts zur Tagung eingereicht und durch die Organisatorinnen und Organisatoren sowie durch den Vorstand der Sektion Medienpädagogik im double 
peer-review Verfahren begutachtet. Die Volltexte wurden nach der Tagung im Herbst 2019 eingereicht und beinhalten daher auch die Rückmeldungen aus den Diskussionen während der Tagung. Um diese Diskussionen weiterzuführen haben die Autorinnen und Autoren eines jeweiligen Tagungspanels ihre Beiträge in einem eher offenen double peer-review Verfahren gegenseitig begutachtet.

\section{Literatur}

Bundesministerium für Bildung, Wissenschaft und Forschung. 2017. «Schule 4.O. - jetzt wird's digital». 2017. https://bildung.bmbwf.gv.at/schulen/schule40/ index.html.

Brinda, Torsten, Ira Diethelm, Rainer Gemulla, Ralf Romeike, Johannes Schöning, Carsten Schulte, und et al. 2016. «Dagstuhl-Erklärung: Bildung in der digitalen vernetzten Welt». https://www.gi.de/aktuelles/meldungen/detailansicht/ article/dagstuhl-erklaerung-bildung-in-der-digitalen-vernetzten-welt.html.

Brinda, Torsten, Niels Brüggen, Ira Diethelm, Thomas Knaus, Sven Kommer, Christine Kopf, Petra Missomelius, Rainer Leschke, Friederike Tilemann, und Andreas Weich. 2019. «Frankfurt-Dreieck zur Bildung in der digital vernetzten Welt. Ein interdisziplinäres Modell». Gesellschaft für Informatik. https:// dagstuhl.gi.de/fileadmin/GI/Allgemein/PDF/Frankfurt-Dreieck-zur-Bildungin-der-digitalen-Welt.pdf.

Deutschschweizer Erziehungsdirektoren-Konferenz (D-EDK), Hrsg. 2014. «Lehrplan 21: Medien und Informatik». D-EDK. http://v-ef.lehrplan.ch/container/V_ EF_DE_Modul_MI.pdf.

KMK - Kultusministerkonferenz. 2016. «Bildung in der digitalen Welt. Strategie der Kultusministerkonferenz». Herausgegeben von Sekretariat der Kultusministerkonferenz. Kultusministerkonferenz. https://www.kmk.org/fileadmin/ Dateien/pdf/PresseUndAktuelles/2017/Digitalstrategie_KMK_Weiterbildung. pdf.

Rummler, Klaus, und Ilka Koppel, Hrsg. 2019. Lernen mit und über Medien in einer digitalen Welt. Herbsttagung der Sektion Medienpädagogik (DGfE). Book of Abstracts. Zürich: Pädagogische Hochschule Zürich. https://doi.org/10.5281/ zenodo.3413860. 


\section{Beiträge}

Altenrath, Maike, Christian Helbig, und Sandra Hofhues. 2020. «Deutungshoheiten: Digitalisierung und Bildung in Programmatiken und Förderrichtlinien Deutschlands und der EU». Herausgegeben von Klaus Rummler, Ilka Koppel, Sandra Aßmann, Patrick Bettinger, und Karsten D. Wolf. MedienPädagogik: Zeitschrift für Theorie und Praxis der Medienbildung, Jahrbuch Medienpädagogik, 17 (Jahrbuch Medienpädagogik): 565-94. https://doi.org/10.21240/mpaed/ jb17/2020.05.22.X.

Autenrieth, Daniel, Claudia Baumbusch, und Anja Marquardt. 2020. «Lehren und Lernen mit und über Medien in Kooperation von Schule, Hochschule und Museen: Am Beispiel des Projekts 〈Reuchlin digital»». Herausgegeben von Klaus Rummler, Ilka Koppel, Sandra Aßmann, Patrick Bettinger, und Karsten D. Wolf. MedienPädagogik: Zeitschrift für Theorie und Praxis der Medienbildung, Jahrbuch Medienpädagogik, 17 (Jahrbuch Medienpädagogik): 531-63. https:// doi.org/10.21240/mpaed/jb17/2020.05.21.X.

Bettinger, Patrick, Saskia Draheim, Simon Meier, und Ellen Witte. 2020. «Making the subject: Eine materiell-diskursive Perspektive auf Lernprozesse in Makerspaces und FabLabs». Herausgegeben von Klaus Rummler, Ilka Koppel, Sandra Aßmann, Patrick Bettinger, und Karsten D. Wolf. MedienPädagogik: Zeitschrift für Theorie und Praxis der Medienbildung, Jahrbuch Medienpädagogik, 17 (Jahrbuch Medienpädagogik): 617-45. https://doi.org/10.21240/mpaed/ jb17/2020.05.24.X.

Böhme, Richard, und Meike Munser-Kiefer. 2020. «Lernunterstützung mit digitalen Unterrichtsmaterialien: Interdisziplinäre Erkenntnisse und Entwicklungsperspektiven». Herausgegeben von Klaus Rummler, Ilka Koppel, Sandra Aßmann, Patrick Bettinger, und Karsten D. Wolf. MedienPädagogik: Zeitschrift für Theorie und Praxis der Medienbildung, Jahrbuch Medienpädagogik, 17 (Jahrbuch Medienpädagogik): 427-54. https://doi.org/10.21240/mpaed/ jb17/2020.05.17.X.

Buchner, Josef, und Diane Aretz. 2020. «Lernen mit immersiver Virtual Reality: Didaktisches Design und Lessons Learned». Herausgegeben von Klaus Rummler, Ilka Koppel, Sandra Aßmann, Patrick Bettinger, und Karsten D. Wolf. MedienPädagogik: Zeitschrift für Theorie und Praxis der Medienbildung, Jahrbuch Medienpädagogik, 17 (Jahrbuch Medienpädagogik): 195-216. https:// doi.org/10.21240/mpaed/jb17/2020.05.01.X.

Gallner-Holzmann, Katharina. 2020. «Visualisierung und Didaktisierung digitaler Archivbestände: Perspektiven zur Gestaltung offener Lernräume für historisches Lernen». Herausgegeben von Klaus Rummler, Ilka Koppel, Sandra Aßmann, Patrick Bettinger, und Karsten D. Wolf. MedienPädagogik: Zeitschrift für Theorie und Praxis der Medienbildung, Jahrbuch Medienpädagogik, 17 (Jahrbuch Medienpädagogik): 373-99. https://doi.org/10.21240/mpaed/ jb17/2020.05.15.X. 
Kerres, Michael. 2020. "Bildung in der digitalen Welt: Über Wirkungsannahmen und die soziale Konstruktion des Digitalen». Herausgegeben von Klaus Rummler, Ilka Koppel, Sandra Aßmann, Patrick Bettinger, und Karsten D. Wolf. MedienPädagogik: Zeitschrift für Theorie und Praxis der Medienbildung, Jahrbuch Medienpädagogik, 17 (Jahrbuch Medienpädagogik): 1-32. https://doi. org $/ 10.21240 / \mathrm{mpaed} / \mathrm{jbl} / 2020.04 .24$.X.

Kiesler, Natalie. 2020. «Medienkompetenzförderung im Lehramtsstudium der Goethe-Universität: Ein Projektbericht zur erfolgreichen Medienproduktion am Beispiel studentischer Erklärvideos». Herausgegeben von Klaus Rummler, Ilka Koppel, Sandra Aßmann, Patrick Bettinger, und Karsten D. Wolf. MedienPädagogik: Zeitschrift für Theorie und Praxis der Medienbildung, Jahrbuch Medienpädagogik, 17 (Jahrbuch Medienpädagogik): 477-506. https://doi. org/10.21240/mpaed/jb17/2020.05.19.X.

Kunkel, Carmen, und Markus Peschel. 2020. «Lernen mit und über digitale Medien im Sachunterricht. Entwicklung eines vielperspektivischen Konzepts zur Erschliessung digitaler Medien». Herausgegeben von Klaus Rummler, Ilka Koppel, Sandra Aßmann, Patrick Bettinger, und Karsten D. Wolf. MedienPädagogik: Zeitschrift für Theorie und Praxis der Medienbildung, Jahrbuch Medienpädagogik, 17 (Jahrbuch Medienpädagogik): 455-76. https://doi.org/10.21240/ mpaed/jb17/2020.05.18.X.

Mayrberger, Kerstin. 2020. «Partizipative Mediendidaktik: Darstellung von Eckpunkten und Vertiefung des Partizipationsraums als konstituierendes Strukturelement». Herausgegeben von Klaus Rummler, Ilka Koppel, Sandra Aßmann, Patrick Bettinger, und Karsten D. Wolf. MedienPädagogik: Zeitschrift für Theorie und Praxis der Medienbildung, Jahrbuch Medienpädagogik, 17 (Jahrbuch Medienpädagogik): 59-92. https://doi.org/10.21240/mpaed/jb17/2020.04.26.X.

Mayrberger, Kerstin, und Kathrin Galley. 2020. «Tablets an Beruflichen Gymnasien: Gelingensfaktoren für die Integration mobiler Endgeräte im Schulunterricht: Ausgewählte Ergebnisse des Projekts 〈tabletBS〉 2015-2019». Herausgegeben von Klaus Rummler, Ilka Koppel, Sandra Aßmann, Patrick Bettinger, und Karsten D. Wolf. MedienPädagogik: Zeitschrift für Theorie und Praxis der Medienbildung, Jahrbuch Medienpädagogik, 17 (Jahrbuch Medienpädagogik): 323-46. https://doi.org/10.21240/mpaed/jb17/2020.05.13.X.

Moser, Heinz. 2021. «Überlegungen zum Lernen mit und über Medien im Zeitalter der Digitalisierung». Herausgegeben von Klaus Rummler, Ilka Koppel, Sandra Aßmann, Patrick Bettinger, und Karsten D. Wolf. MedienPädagogik: Zeitschrift für Theorie und Praxis der Medienbildung, Jahrbuch Medienpädagogik, 17 (Jahrbuch Medienpädagogik): 709-32. https://doi.org/10.21240/mpaed/ jb17/2021.05.18.X.

Niesyto, Horst, und Thorsten Junge. 2020. «Digitale Medien in der Grundschullehrerbildung: Erfahrungen aus dem Projekt dileg-SL». Herausgegeben von Klaus Rummler, Ilka Koppel, Sandra Aßmann, Patrick Bettinger, und Karsten D. Wolf. MedienPädagogik: Zeitschrift für Theorie und Praxis der Medienbildung, Jahrbuch Medienpädagogik, 17 (Jahrbuch Medienpädagogik): 297-322. https://doi.org/10.21240/mpaed/jb17/2020.05.12.X. 
Philipp, Maik. 2020. «Leseförderung 4.0?: Gibt es Unterschiede in den Merkmalen effektiver Lesefördermassnahmen mit multiplen Dokumenten, wenn digitale Medien genutzt werden?» Herausgegeben von Klaus Rummler, Ilka Koppel, Sandra Aßmann, Patrick Bettinger, und Karsten D. Wolf. MedienPädagogik: Zeitschrift für Theorie und Praxis der Medienbildung, Jahrbuch Medienpädagogik, 17 (Jahrbuch Medienpädagogik): 141-68. https://doi.org/10.21240/mpaed/ jb17/2020.04.29.X.

Rau, Franco. 2020. «Lernen mit und über Wikibooks: Erkenntnisse entwicklungsorientierter Fallstudien zur integrativen Medienbildung im Lehramtsstudium». Herausgegeben von Klaus Rummler, Ilka Koppel, Sandra Aßmann, Patrick Bettinger, und Karsten D. Wolf. MedienPädagogik: Zeitschrift für Theorie und Praxis der Medienbildung, Jahrbuch Medienpädagogik, 17 (Jahrbuch Medienpädagogik): 273-96. https://doi.org/10.21240/mpaed/jb17/2020.05.11.X.

Repenning, Alexander, Nora Anna Escherle, und Anna Lamprou. 2020. «Die ersten 1000: Computational Thinking als obligatorische Ausbildung für Primarschullehrpersonen in der Schweiz». Herausgegeben von Klaus Rummler, Ilka Koppel, Sandra Aßmann, Patrick Bettinger, und Karsten D. Wolf. MedienPädagogik: Zeitschrift für Theorie und Praxis der Medienbildung, Jahrbuch Medienpädagogik, 17 (Jahrbuch Medienpädagogik): 595-616. https://doi.org/10.21240/ mpaed/jb17/2020.05.23.X.

Scheidig, Falk. 2020. «Lehren lernen mit digitalen Medien: Technologiegestützte Praxisbezüge in der Lehrpersonenbildung». Herausgegeben von Klaus Rummler, Ilka Koppel, Sandra Aßmann, Patrick Bettinger, und Karsten D. Wolf. MedienPädagogik: Zeitschrift für Theorie und Praxis der Medienbildung, Jahrbuch Medienpädagogik, 17 (Jahrbuch Medienpädagogik): 675-708. https://doi.org/10.21240/mpaed/jb17/2020.05.26.X.

Schepkowski, David, Martin Burghardt, und Dr. Peter Ferdinand. 2020. «Pädagogisch-Didaktische Herausforderungen bei der Entwicklung von digitalen Lernumgebungen in der laborbasierten Lehre: Am Beispiel des Projekts 〈DigiLab4You»». Herausgegeben von Klaus Rummler, Ilka Koppel, Sandra Aßmann, Patrick Bettinger, und Karsten D. Wolf. MedienPädagogik: Zeitschrift für Theorie und Praxis der Medienbildung, Jahrbuch Medienpädagogik, 17 (Jahrbuch Medienpädagogik): 347-72. https://doi.org/10.21240/mpaed/jb17/2020.05.14.X.

Schlote, Elke, Daniel Klug, und Klaus Neumann-Braun. 2020. «Mittendrin statt nur dabei: Partizipation im schulischen Unterricht mit der Web-App TRAVIS GO digital unterstützen». Herausgegeben von Klaus Rummler, Ilka Koppel, Sandra Aßmann, Patrick Bettinger, und Karsten D. Wolf. MedienPädagogik: Zeitschrift für Theorie und Praxis der Medienbildung, Jahrbuch Medienpädagogik, 17 (Jahrbuch Medienpädagogik): 507-29. https://doi.org/10.21240/mpaed/ jbl7/2020.05.20.X.

Schmid, Mirjam, und Dominik Petko. 2020. «Technological Pedagogical Content Knowledge> als Leitmodell medienpädagogischer Kompetenz». Herausgegeben von Klaus Rummler, Ilka Koppel, Sandra Aßmann, Patrick Bettinger, und Karsten D. Wolf. MedienPädagogik: Zeitschrift für Theorie und Praxis der Medienbildung, Jahrbuch Medienpädagogik, 17 (Jahrbuch Medienpädagogik): 121-40. https://doi.org/10.21240/mpaed/jb17/2020.04.28.X. 
Schöb, Sabine. 2020. «Lernen mit Medien - (k)ein nachhaltiger Ansatz für die Kompetenzentwicklung Lehrender im Kontext der Erwachsenenbildung/ Weiterbildung?!» Herausgegeben von Klaus Rummler, Ilka Koppel, Sandra Aßmann, Patrick Bettinger, und Karsten D. Wolf. MedienPädagogik: Zeitschrift für Theorie und Praxis der Medienbildung, Jahrbuch Medienpädagogik, 17 (Jahrbuch Medienpädagogik): 647-74. https://doi.org/10.21240/mpaed/jb17/2020.05.25.X.

Sick, Christina. 2020. "Veränderung von Einstellungen und Verhaltensdeterminanten mittels videobasierter Narrationen mit parallelen Erklärungen». Herausgegeben von Klaus Rummler, Ilka Koppel, Sandra Aßmann, Patrick Bettinger, und Karsten D. Wolf. MedienPädagogik: Zeitschrift für Theorie und Praxis der Medienbildung, Jahrbuch Medienpädagogik, 17 (Jahrbuch Medienpädagogik): 245-71. https://doi.org/10.21240/mpaed/jb17/2020.05.03.X.

Swertz, Christian. 2020. «Big Data als datenbasierte Programmierung: Eine medienpädagogische Analyse künstlicher neuronaler Netzwerke». Herausgegeben von Klaus Rummler, Ilka Koppel, Sandra Aßmann, Patrick Bettinger, und Karsten D. Wolf. MedienPädagogik: Zeitschrift für Theorie und Praxis der Medienbildung, Jahrbuch Medienpädagogik, 17 (Jahrbuch Medienpädagogik): 93-119. https://doi.org/10.21240/mpaed/jb17/2020.04.27.X.

Thumel, Mareike, Anja Schwedler-Diesener, Steffen Greve, Jessica Süßenbach, Florian Jastrow, und Claus Krieger. 2020. "Inszenierungsmöglichkeiten eines mediengestützten Sportunterrichts». Herausgegeben von Klaus Rummler, Ilka Koppel, Sandra Aßmann, Patrick Bettinger, und Karsten D. Wolf. MedienPädagogik: Zeitschrift für Theorie und Praxis der Medienbildung, Jahrbuch Medienpädagogik, 17 (Jahrbuch Medienpädagogik): 401-26. https://doi. org/10.21240/mpaed/jb17/2020.05.16.X.

Totter, Alexandra, Julia Häbig, Daniela Müller-Kuhn, und Enikö Zala-Mezö. 2020. «Zwischen traditionellem Schulbuch und hybridem Lehrmittel: Bedingungen, Möglichkeiten und Herausforderungen von Lernen und Lehren in einer digitalen Welt». Herausgegeben von Klaus Rummler, Ilka Koppel, Sandra Aßmann, Patrick Bettinger, und Karsten D. Wolf. MedienPädagogik: Zeitschrift für Theorie und Praxis der Medienbildung, Jahrbuch Medienpädagogik, 17 (Jahrbuch Medienpädagogik): 169-93. https://doi.org/10.21240/mpaed/jb17/2020.04.30.X.

Wedler, Katharina. 2020. «Selbstreferentialität und Historisierung als Werkzeuge medialen Handelns im bildungswissenschaftlichen Kontext: Zum Potenzial populärer Kultur im Erarbeitungsprozess erklärender Kurzfilme». Herausgegeben von Klaus Rummler, Ilka Koppel, Sandra Aßmann, Patrick Bettinger, und Karsten D. Wolf. MedienPädagogik: Zeitschrift für Theorie und Praxis der Medienbildung, Jahrbuch Medienpädagogik, 17 (Jahrbuch Medienpädagogik): 217-43. https://doi.org/10.21240/mpaed/jb17/2020.05.02.X.

Wienberg, Jana, und Klaus Buddeberg. 2020. «Resonanzstrategien im Umgang mit Beschleunigung und Digitalisierung». Herausgegeben von Klaus Rummler, Ilka Koppel, Sandra Aßmann, Patrick Bettinger, und Karsten D. Wolf. MedienPädagogik: Zeitschrift für Theorie und Praxis der Medienbildung, Jahrbuch Medienpädagogik, 17 (Jahrbuch Medienpädagogik): 33-58. https://doi. org/10.21240/mpaed/jb17/2020.04.25.X. 